\title{
DENSITY PROBLEMS ON VECTOR BUNDLES AND MANIFOLDS
}

\author{
LASHI BANDARA
}

(Communicated by James E. Colliander)

\begin{abstract}
We study some canonical differential operators on vector bundles over smooth, complete Riemannian manifolds. Under very general assumptions, we show that smooth, compactly supported sections are dense in the domains of these operators. Furthermore, we show that smooth, compactly supported functions are dense in second order Sobolev spaces on such manifolds under the sole additional assumption that the Ricci curvature is uniformly bounded from below.
\end{abstract}

\section{INTRODUCTION}

In the analysis of differential operators, it is often useful in calculations to know that smooth, compactly supported functions are dense in the domain of the operator in question. We call this the density problem. In this paper, we show that the density problem can be solved in the positive for some canonical differential operators over a wide class of vector bundles.

More precisely, let $\mathcal{V}$ be a smooth vector bundle over a smooth, complete Riemannian manifold $\mathcal{M}$. Suppose that $\mathcal{V}$ is equipped with a metric $\mathrm{h}$ and connection $\nabla$ that are compatible. Defining div $=-\nabla^{*}$ in the $\mathrm{L}^{2}$ theory, we show that $\mathrm{C}_{\mathrm{c}}^{\infty}\left(\mathrm{T}^{*} \mathcal{M} \otimes \mathcal{V}\right)$ is dense in $\mathcal{D}($ div $)$. Furthermore, letting $\Delta_{\mathrm{B}}=-\operatorname{div} \bar{\nabla}$, the Bochner Laplacian on $\mathcal{V}$, we show that $\mathrm{C}_{\mathrm{c}}^{\infty}(\mathcal{V})$ is dense in $\mathcal{D}\left(\Delta_{\mathrm{B}}\right)$. In the case that $\mathcal{V}=\boldsymbol{\Omega}(\mathcal{M})$, the exterior algebra over $\mathcal{M}$, we consider the operator $\mathrm{d}$, the exterior derivative, and its adjoint $\delta=\mathrm{d}^{*}$. In this situation, we show that $\mathrm{C}_{\mathrm{c}}^{\infty}(\boldsymbol{\Omega}(\mathcal{M}))$ is dense in $\mathcal{D}(\delta)$.

While some of the results we present in this paper are known and can be accessed via alternative methods, our result on the density problem for second order Sobolev spaces on manifolds is new. The Sobolev space $\mathrm{W}^{2,2}(\mathcal{M})$ is defined as the closure of functions $u \in \mathrm{C}^{\infty} \cap \mathrm{L}^{2}(\mathcal{M})$ satisfying $|\nabla u|,\left|\nabla^{2} u\right| \in \mathrm{L}^{2}(\mathcal{M})$ with respect to the norm $\|u\|_{\mathrm{W}^{2,2}}=\|u\|+\|\nabla u\|+\left\|\nabla^{2} u\right\|$, and $\mathrm{W}_{0}^{2,2}(\mathcal{M})$ as the closure of $\mathrm{C}_{\mathrm{c}}^{\infty}(\mathcal{M})$ under the same norm. According to Hebey in [7, the best known conditions yielding $\mathrm{W}_{0}^{2,2}(\mathcal{M})=\mathrm{W}^{2,2}(\mathcal{M})$ require both $\operatorname{Ric} \geq \eta \mathrm{g}$ and $\operatorname{inj}(\mathcal{M}) \geq \kappa$, for some $\eta \in \mathbb{R}$ and $\kappa>0$. We dispense with the latter bound, yielding the following highlighted theorem of this paper.

Received by the editors August 20, 2012.

2010 Mathematics Subject Classification. Primary 46E35, 53C21, 58J60.

Key words and phrases. Density problems, first order operators on vector bundles, Laplacian on vector bundles, second order Sobolev spaces on manifolds. 
Theorem 1.1. Let $\mathcal{M}$ be a smooth, complete Riemannian manifold with metric $\mathrm{g}$ and Levi-Cevita connection $\nabla$. If there exists $\eta \in \mathbb{R}$ such that $\mathrm{Ric} \geq \eta \mathrm{g}$, then $\mathrm{W}_{0}^{2,2}(\mathcal{M})=\mathrm{W}^{2,2}(\mathcal{M})$

Our motivation to study density problems emerges from the study of Kato square root type problems in the presence of geometry. The classical version of this problem on $\mathbb{R}^{n}$ was solved by Auscher, Hofmann, Lacy, McIntosh and Tchamitchian in [1] and was rephrased in a first order point of view in [3] by Axelsson (Rosén), Keith and McIntosh. The proofs in the latter paper particularly exploit the density of compactly supported smooth functions and vector fields in the domains of the gradient and divergence operators respectively. Morris in [9] combines ideas from [3] and [2], and rephrases and solves similar questions in the context of submanifolds in $\mathbb{R}^{n}$. There, density facts were needed, but these were handled by different techniques. In formulating and solving a Kato square root problem on vector bundles by the author and McIntosh in 4], density problems became of central importance. While some of these issues were circumvented by alternative means, the desire to address density concerns persisted.

The main theme and philosophy in this paper are the following. Given a first order differential operator $D: \mathrm{C}^{\infty}(\mathcal{V}) \rightarrow \mathrm{C}^{\infty}(\mathcal{W})$ (where $\mathcal{W}$ is another vector bundle), we construct an operator $\Pi$ that is symmetric on $\mathrm{C}_{\mathrm{c}}^{\infty}(\mathcal{V}) \oplus \mathrm{C}_{\mathrm{c}}^{\infty}(\mathcal{W})$ such that it encodes $D$ in a natural way. We then show that the density results follow from, or are sometimes equivalent to, showing that $\Pi$ is essentially self-adjoint. The density problem for second order Sobolev spaces on manifolds follows from the essential self-adjointness of a particular $\Pi^{2}$ coupled with the lower bound on Ricci curvature. The paper [5] by Chernoff, introduced to the author by Baskin, justifies this reduction of density problems to essential self-adjointness, as it provides a set of very general conditions under which symmetric operators and their powers are essentially self-adjoint.

\section{Preliminaries}

2.1. Notation. Throughout this paper, we assume Einstein summation notation. Explicitly, whenever there is a raised and lowered index appearing multiplicatively, we assume summation over that index. For two quantities $a, b \geq 0$, we express inequalities up to a constant by writing $a \lesssim b$. By this we mean that there exists a $C>0$ such that $a \leq C b$. The constant $C$ will be independent of the quantities $a$ and $b$, and the dependence will be clear from context or preceding hypotheses. By writing $a \simeq b$, we mean that $a \lesssim b$ and $b \lesssim a$.

2.2. Operator theory. In this section, we provide an exposition of ideas from operator theory that we use in this paper. While some of the ideas here are valid for operators on general Banach spaces, we restrict ourselves to the theory in Hilbert spaces. We refer the reader to the excellent books [8] by Kato and [1] by Yosida which provide a more complete description of operator theory.

Let $\mathscr{H}_{1}$ and $\mathscr{H}_{2}$ be Hilbert spaces with inner products $\langle\cdot, \cdot\rangle_{j}: \mathscr{H}_{j} \times \mathscr{H}_{j} \rightarrow \mathbb{C}$. We say that a linear map $T: \mathcal{D}(T) \subset \mathscr{H}_{1} \rightarrow \mathscr{H}_{2}$ is an operator with domain $\mathcal{D}(T)$. If $S$ is an operator such that $\mathcal{D}(S) \subset \mathcal{D}(T)$ and $T u=S u$ for $u \in \mathcal{D}(S)$, then we write $S \subset T$ and say that $T$ extends $S$. We emphasise that an operator is characterised by both the map and the domain. 
An operator $T$ is said to be densely-defined if $\overline{\mathcal{D}(T)}=\mathscr{H}_{1}$, and it is said to be closed if its graph, $\mathscr{G}(T)=\{(u, T u): u \in \mathcal{D}(T)\}$, is a closed subset of $\mathscr{H}_{1} \times \mathscr{H}_{2}$. The latter notion is equivalent to requiring that whenever $u_{n} \in \mathcal{D}(T)$ such that $u_{n} \rightarrow u \in \mathscr{H}_{1}$ and $T u_{n} \rightarrow v \in \mathscr{H}_{2}$, then $u \in \mathcal{D}(T)$ and $T u=v$. Define the operator norm of $T$ by $\|u\|_{T}=\|u\|_{\mathscr{H}_{1}}+\|T u\|_{\mathscr{H}_{2}}$ whenever $u \in \mathcal{D}(T)$. Then, an operator $T$ is closed if and only if $\left(\mathcal{D}(T),\|\cdot\|_{T}\right)$ is a Banach space. When we say " $X$ is dense in $\mathcal{D}(T)$," we mean that $X \subset \mathcal{D}(T)$ is dense in the operator norm of $T$. We make a motivational remark that the notions closed and densely-defined are particularly useful when studying differential operators.

By the closed graph theorem (see Theorem 5.20 in [8]), a closed operator $T$ with $\mathcal{D}(T)=\mathscr{H}$ is bounded, by which we mean there exists $C>0$ such that $\|T u\| \leq C\|u\|$ for $u \in \mathscr{H}$. Boundedness of an operator is equivalent to saying that it is continuous.

A notion that will be very important in later parts is that an operator $T$ be closable. By this we mean that $\overline{\mathscr{G}(T)}$ is equal to the graph of another operator $\bar{T}$ called the closure of $T$. An operator $T$ is closable if and only if $u_{n} \in \mathcal{D}(T)$ with $u_{n} \rightarrow 0$ and $T u_{n} \rightarrow v$ implies $v=0$. It is immediate that $T \subset \bar{T}$.

We say that an operator $S: \mathcal{D}(S) \subset \mathscr{H}_{2} \rightarrow \mathscr{H}_{1}$ is adjoint to $T$ if $\langle T u, v\rangle_{\mathscr{H}_{2}}=$ $\langle u, S v\rangle_{\mathscr{H}_{1}}$ for all $u \in \mathcal{D}(T)$ and $v \in \mathcal{D}(S)$. For such operators, if one is denselydefined, then the other is closable (see Theorem 5.28 in [8]). This is an important fact that we often use. For a given $T$, there are many operators $S$ that are adjoint to $T$. However, if $T$ is densely-defined, then there exists a unique maximal operator $T^{*}$ adjoint to $T$ called the adjoint of $T$. By maximal, we mean that if $S$ is any operator adjoint to $T$, then $S \subset T^{*}$. We construct $T^{*}$ in the following way. Let $v \in \mathcal{D}\left(T^{*}\right)$ if there exists $f \in \mathscr{H}_{1}$ such that $\langle T u, v\rangle_{\mathscr{H}_{2}}=\langle u, f\rangle_{\mathscr{H}_{1}}$ for all $u \in \mathcal{D}(T)$ and define $T^{*} v=f$. The uniqueness of $T^{*}$ follows since $\mathcal{D}(T)$ is dense in $\mathscr{H}$. If further $T$ is closable, then $T^{*}$ is densely-defined and closed and, furthermore, $T^{* *}=\bar{T}$ (see Theorem 5.29 in 8 ).

An important situation arises when $\mathscr{H}=\mathscr{H}_{1}=\mathscr{H}_{2}$. There, we say an operator $T$ is hermitian symmetric (or simply symmetric) to mean $\langle T u, v\rangle=\langle u, T v\rangle$, for all $u, v \in \mathcal{D}(T)$. The operator $T$ is said to be skew-symmetric if $\langle T u, v\rangle=\langle u,-T v\rangle$. A symmetric operator is said to be self-adjoint if $T^{*}=T$. Explicitly, this means $T u=T^{*} u$ for all $u \in \mathcal{D}(T)=\mathcal{D}\left(T^{*}\right)$. Note that a self-adjoint operator is necessarily densely-defined and closed. A densely-defined, closable operator $T$ is said to be essentially self-adjoint if $\bar{T}$ is self-adjoint. Self-adjointness will be one of the primary tools that we use in this paper. Although we run the risk of labouring the point, we present the following description of the maximal nature of self-adjointness.

Proposition 2.1. Let $T$ be self-adjoint, and $S$ a symmetric extension of $T$. Then $S=T$.

Proof. First, since $T=S$ on $\mathcal{D}(T)$, it suffices to prove that $\mathcal{D}(S) \subset \mathcal{D}(T)$. By the fact that $S$ is symmetric, we have that for all $u, v \in \mathcal{D}(S),\langle S u, v\rangle=\langle u, S v\rangle$. In particular, this reads as $\langle S u, v\rangle=\langle u, T v\rangle$ whenever $v \in \mathcal{D}(T)$. By the construction of the adjoint operator, we conclude that $u \in \mathcal{D}\left(T^{*}\right)$ and that $T^{*} u=S u$. But by the self-adjointness of $T, \mathcal{D}\left(T^{*}\right)=\mathcal{D}(T)$, and so we have that $\mathcal{D}(S) \subset \mathcal{D}(T)$.

The following proposition yields the same conclusion as above, but it is more useful since we only need to verify that the operators are equal on a dense subset. 
Proposition 2.2. Let $T$ and $S$ be self-adjoint. If $\mathcal{D} \subset \mathcal{D}(T) \cap \mathcal{D}(S), \mathcal{D}$ is dense in $\mathcal{D}(T)$, and $T u=S u$ for all $u \in \mathcal{D}$, then $T=S$.

Proof. Since $S$ is self-adjoint, in particular it is symmetric, and thus, by Proposition 2.1 it suffices to show that $T \subset S$.

Let $u \in \mathcal{D}(T)$. By the hypotheses, there exists a sequence $u_{j} \in \mathcal{D}$ such that $u_{j} \rightarrow u$ and $T u_{j} \rightarrow T u$, and further $T u_{j}=S u_{j}$. Thus, we have that $u_{j} \rightarrow u$ and $S u_{j} \rightarrow T u$. But the self-adjointness of $S$ in particular means that $S$ is closed, and hence $u \in \mathcal{D}(S)$ and $S u=T u$. This shows that $T \subset S$ as required.

2.3. Hyperbolic equations and essential self-adjointness. The work of Chernoff in [5] is central to proving the results we present in this paper. Thus, for the convenience of the reader, we recall some notions from this paper.

Our setting is the following. Let $\mathcal{M}$ be a smooth, complete Riemannian manifold with smooth metric g and volume measure $d \mu$. Further, let $\mathcal{V}$ denote a smooth, complex vector bundle of finite rank over $\mathcal{M}$ with smooth metric $\mathrm{h}$. Since $\mathcal{V}$ is complex, we assume that $\mathrm{h}$ is hermitian. We denote the fibre of $\mathcal{V}$ over $x \in \mathcal{M}$ by $\mathcal{V}_{x}$. When we consider real bundles, we always implicitly identify them under their complexification.

By $\boldsymbol{\Gamma}(\mathcal{V})$ denote the $d \mu$-measurable sections of $\mathcal{V}$. That is, $\xi \in \boldsymbol{\Gamma}(\mathcal{V})$ if $\xi: \mathcal{M} \rightarrow \mathcal{V}$, $\xi(x) \in \mathcal{V}_{x}$ and $d \mu$-measurable in $x$. Then, we define $\mathrm{L}^{2}(\mathcal{V})$ as the space of $\xi \in \boldsymbol{\Gamma}(\mathcal{V})$ such that

$$
\int_{\mathcal{M}}|\xi(x)|_{x}^{2} d \mu(x)<\infty
$$

We note that $\mathrm{L}^{2}(\mathcal{V})$ is a Hilbert space equipped with the inner product

$$
\langle\xi, \eta\rangle=\int_{\mathcal{M}} \mathrm{h}(\xi(x), \eta(x))_{x} d \mu(x) .
$$

Next, let $L$ be a first order differential operator on $\mathrm{C}^{\infty}(\mathcal{V})$, and let $L_{c}=L$ on $\mathrm{C}_{\mathrm{c}}^{\infty}(\mathcal{V})$. We recall the symbol of $L$ from [5. Let $x \in \mathcal{M}, v \in \mathrm{T}_{x}^{*} \mathcal{M}, e \in \mathcal{V}_{x}$ and fix $g \in \mathrm{C}^{\infty}(\mathcal{M})$ and $f \in \mathrm{C}^{\infty}(\mathcal{V})$ such that $\mathrm{d} g(x)=v$ and $f(x)=e$. Then, the symbol of $L$ at $x$ in the direction $v$ acting on $e$ is defined by

$$
\sigma(x, v) e=\sigma_{L}(x, v) e=[L, g I] f(x) .
$$

We note that this is really the principal symbol of the operator $L$ and emphasise as in [5] that this definition only applies to first order operators.

The speed of propagation at $x$ is given by

$$
\mathrm{c}(x)=\mathrm{c}_{L}(x)=\sup _{|v|=1}|\sigma(x, v)|,
$$

where $|\sigma(x, v)|=\sup _{|e|=1}|\sigma(x, v) e|$. Fix $x_{0} \in \mathcal{M}$ and $r>0$. Then, the speed of propagation of $L$ inside the ball $B\left(x_{0}, r\right)$ is given by

$$
\mathrm{c}(r)=\mathrm{c}_{L}(r)=\sup _{x \in B\left(x_{0}, r\right)} \mathrm{c}(x) .
$$

With this notation in hand, we present the following theorem, which is an immediate consequence of Theorem 2.2 in [5]. This result is the central tool that we use in this paper.

Theorem 2.3. Let $\mathcal{V}$ be a smooth vector bundle with smooth metric $\mathrm{h}$ over a smooth, complete Riemannian manifold $\mathcal{M}$. Let $\Pi$ be a first order differential operator on $\mathrm{C}^{\infty}(\mathcal{V})$ such that $\Pi_{c}=\Pi$ with domain $\mathrm{C}_{\mathrm{c}}^{\infty}(\mathcal{V})$ is symmetric. Furthermore, 
suppose that there exists $C>0$ such that $\mathrm{c}_{\Pi}(x) \leq C$ for each $x \in \mathcal{M}$. Then, every power of $\Pi_{c}$ is essentially self-adjoint.

Proof. We apply Theorem 2.2 in [5] which states that if $L$ is a skew-symmetric operator on $\mathrm{C}_{\mathrm{c}}^{\infty}(\mathcal{V})$ and $\int_{0}^{\infty} \mathrm{c}_{L}(r)^{-1} d r=+\infty$, then every power of $-\imath L$ is essentially self-adjoint.

First, let $L=\imath \Pi$. Then, it is easy to see that $L_{c}=L$ with domain $\mathcal{D}\left(L_{c}\right)=$ $\mathrm{C}_{\mathrm{c}}^{\infty}(\mathcal{V})$ is skew-symmetric. Furthermore, an easy calculation yields that $\sigma_{L}(x, v) e=$ $\imath \sigma_{\Pi}(x, v) e$. Therefore, $\left|\sigma_{L}(x, v) e\right|=\left|\sigma_{\Pi}(x, v) e\right|$, which implies that $\mathrm{c}_{L}(r)=\mathrm{c}_{\Pi}(r) \leq$ $C$ after fixing a base point $x_{0} \in \mathcal{M}$. Thus,

$$
\int_{0}^{\infty} \frac{d r}{\mathrm{c}_{L}(r)}=\int_{0}^{\infty} \frac{d r}{\mathrm{c}_{\Pi}(r)} \geq \int_{0}^{\infty} \frac{d r}{C}=+\infty .
$$

Putting these facts together demonstrates that $L$ satisfies the hypotheses of Theorem 2.2 in [5], and therefore we conclude that every power of the operator $\Pi_{c}$ is essentially self-adjoint.

\section{Connections, Divergence and Laplacians on Vector bundles}

In this section, we further assume that $\mathcal{V}$ is equipped with a connection $\nabla$. Recall that this is a map $\nabla: \mathrm{C}^{\infty}(\mathcal{V}) \rightarrow \mathrm{C}^{\infty}\left(\mathrm{T}^{*} \mathcal{M} \otimes \mathcal{V}\right)$ satisfying the Leibniz rule

$$
\nabla(f X)=\nabla f \otimes X+f \nabla X
$$

for all $f \in \mathrm{C}^{\infty}(\mathcal{M}), X \in \mathrm{C}^{\infty}(\mathcal{V})$ and where $\nabla f=\mathrm{d} f$ is the exterior derivative on $\mathcal{M}$. Furthermore, let us assume that $\nabla$ and $\mathrm{h}$ are compatible, by which we mean the product rule

$$
X \mathrm{~h}(Y, Z)=\mathrm{h}\left(\nabla_{X} Y, Z\right)+\mathrm{h}\left(Y, \nabla_{X} Z\right)
$$

holds for all $X \in \mathrm{C}^{\infty}(\mathrm{T} \mathcal{M})$ and $Y, Z \in \mathrm{C}^{\infty}(\mathcal{V})$. By letting $\nabla_{c}$ be the restriction of $\nabla$ to $\mathrm{C}_{\mathrm{c}}^{\infty}(\mathcal{V})$, we find that

$$
\left\langle\nabla_{c} u, v\right\rangle=\left\langle u,-\operatorname{tr} \nabla_{c} v\right\rangle,
$$

for all $u \in \mathrm{C}_{\mathrm{c}}^{\infty}(\mathcal{V})$ and $v \in \mathrm{C}_{\mathrm{c}}^{\infty}\left(\mathrm{T}^{*} \mathcal{M} \otimes \mathcal{V}\right)$ (see Proposition 6.1 in [4] for a proof). This means the operators $\nabla_{c}$ and $-\operatorname{tr} \nabla_{c}$ are adjoint to each other, and by the density of $\mathrm{C}_{\mathrm{c}}^{\infty}$ in $\mathrm{L}^{2}$, both these operators are densely-defined and closable.

Define $-\operatorname{div}=\nabla_{c}{ }^{*}$. Clearly, $\operatorname{tr} \nabla_{c} \subset$ div and, moreover, $\overline{\operatorname{tr} \nabla_{c}} \subset$ div. Furthermore, let $\tilde{\nabla}=\left(-\operatorname{tr} \nabla_{c}\right)^{*}$. It is clear that $\overline{\nabla_{c}} \subset \tilde{\nabla}$. In this section we show that under appropriate conditions, $\overline{\nabla_{c}}=\tilde{\nabla}$. The following proposition illustrates the connection of this statement to density problems.

Proposition 3.1. Let $\mathcal{M}$ be a smooth Riemannian manifold and $\mathcal{V}$ a vector bundle with metric $\mathrm{h}$ and connection $\nabla$ that are compatible. Then, the following are equivalent:

(i) $\overline{\operatorname{tr} \nabla_{c}}=\operatorname{div}$,

(ii) $\overline{\nabla_{c}}=\tilde{\nabla}$,

(iii) $\mathrm{C}_{\mathrm{c}}^{\infty}(\mathcal{V})$ is dense in $\mathcal{D}(\tilde{\nabla})$,

(iv) $\mathrm{C}_{\mathrm{c}}^{\infty}\left(\mathrm{T}^{*} \mathcal{M} \otimes \mathcal{V}\right)$ is dense in $\mathcal{D}(\operatorname{div})$.

Proof. Suppose that $\overline{\operatorname{tr} \nabla_{c}}=$ div. Since $\overline{\nabla_{c}}$ is closed and densely-defined, $(-\operatorname{div})^{*}=$ $\nabla_{c}{ }^{* *}=\overline{\nabla_{c}}$. Also, $(-\operatorname{div})^{*}={\overline{\operatorname{tr} \nabla_{c}}}^{*}=\left(\operatorname{tr} \nabla_{c}\right)^{*}=\tilde{\nabla}$. Thus (i) implies (ii). By similar reasoning, (ii) implies (i). Now, we note that $\overline{\nabla_{c}}=\tilde{\nabla}$ if and only if $\mathrm{C}_{\mathrm{c}}^{\infty}(\mathcal{V})$ is dense in $\mathcal{D}(\tilde{\nabla})$ since $\nabla_{c}=\tilde{\nabla}$ on $\mathrm{C}_{\mathrm{c}}^{\infty}(\mathcal{V})$. Thus, (ii) and (iii) are equivalent. By 
a similar argument, (iv) and (i) are equivalent to each other. This concludes the proof.

We also consider the following self-adjoint operators $\Delta_{\mathrm{B}}=-\operatorname{div} \overline{\nabla_{c}}$ with domain

$$
\mathcal{D}\left(\Delta_{\mathrm{B}}\right)=\left\{u \in \mathrm{L}^{2}(\mathcal{V}): u \in \mathcal{D}\left(\overline{\nabla_{c}}\right), \overline{\nabla_{c}} u \in \mathcal{D}(\operatorname{div})\right\} \subset \mathcal{D}\left(\overline{\nabla_{c}}\right)
$$

and $\Delta_{\mathrm{A}}=-\overline{\operatorname{tr} \nabla_{c}} \tilde{\nabla}$ with domain

$$
\mathcal{D}\left(\Delta_{\mathrm{A}}\right)=\left\{u \in \mathrm{L}^{2}(\mathcal{V}): u \in \mathcal{D}(\tilde{\nabla}), \tilde{\nabla} u \in \mathcal{D}\left(\overline{\operatorname{tr} \nabla_{c}}\right)\right\} \subset \mathcal{D}(\tilde{\nabla}) .
$$

These are two Laplacians on the vector bundle. Furthermore, by (因), we find that the connection Laplacian $\Delta_{c}=-\operatorname{tr} \nabla_{c}^{2}$ with domain $\mathrm{C}_{\mathrm{c}}^{\infty}(\mathcal{V})$ is densely-defined and closable. Let $\Delta_{0}$ denote the closure of $\Delta_{c}$ and denote its domain by $\mathcal{D}\left(\Delta_{0}\right)$. Certainly, it is easy to see that $\Delta_{0} \subset \Delta_{\mathrm{B}}$ and $\Delta_{0} \subset \Delta_{\mathrm{A}}$, and we have the following proposition which lists some equivalences.

Proposition 3.2. Under the same hypotheses as Proposition 3.1, the following are equivalent:

(i) $\Delta_{0}$ is self-adjoint,

(ii) $\Delta_{0}=\Delta_{\mathrm{B}}=\Delta_{\mathrm{A}}$,

(iii) $\mathrm{C}_{\mathrm{c}}^{\infty}(\mathcal{V})$ is dense in $\mathcal{D}\left(\Delta_{\mathrm{B}}\right)$,

(iv) $\mathrm{C}_{\mathrm{c}}^{\infty}(\mathcal{V})$ is dense in $\mathcal{D}\left(\Delta_{\mathrm{A}}\right)$.

Proof. If $\Delta_{0}$ is self-adjoint, then since $\Delta_{0} \subset \Delta_{\mathrm{B}}$ and $\Delta_{0} \subset \Delta_{\mathrm{A}}$ where $\Delta_{\mathrm{B}}$ and $\Delta_{\mathrm{A}}$ are in particular symmetric, Proposition 2.1 allows us to conclude that $\Delta_{0}=\Delta_{\mathrm{B}}$ and $\Delta_{0}=\Delta_{\mathrm{A}}$. Thus, (i) implies (ii). It is easy to see that (ii) implies (iii) since $\Delta_{0}=\Delta_{\mathrm{B}}$ on $\mathrm{C}_{\mathrm{c}}^{\infty}(\mathcal{V})$. Similarly, (ii) implies (iv). If (iv) holds, then $\Delta_{0}=\Delta_{\mathrm{A}}$ and $\Delta_{\mathrm{A}}$ is self-adjoint, and so (iv) implies (i). Similarly, (iii) implies (i), and this concludes the proof.

We also define the following objects that we call co-Laplacians. First, define $\triangleright=-\overline{\nabla_{c}} \operatorname{div}$ with domain

$$
\mathcal{D}(\triangleright)=\left\{u \in \mathrm{L}^{2}\left(\mathrm{~T}^{*} \mathcal{M} \otimes \mathcal{V}\right): u \in \mathcal{D}(\operatorname{div}), \operatorname{div} u \in \mathcal{D}\left(\overline{\nabla_{c}}\right)\right\} \subset \mathcal{D}(\operatorname{div}) .
$$

Also, let $\tilde{\triangleright}=-\tilde{\nabla} \overline{\operatorname{tr} \nabla_{c}}$ with domain

$$
\mathcal{D}(\tilde{\triangleright})=\left\{u \in \mathrm{L}^{2}\left(\mathrm{~T}^{*} \mathcal{M} \otimes \mathcal{V}\right): u \in \mathcal{D}\left(\overline{\operatorname{tr} \nabla_{c}}\right), \overline{\operatorname{tr} \nabla_{c}} u \in \mathcal{D}(\tilde{\nabla})\right\} \subset \mathcal{D}\left(\overline{\operatorname{tr} \nabla_{c}}\right) .
$$

It is easy to see that both these operators are self-adjoint. The corresponding connection co-Laplacian is then given by $\triangleright_{c}$ with domain $\mathrm{C}_{\mathrm{c}}^{\infty}\left(\mathrm{T}^{*} \mathcal{M} \otimes \mathcal{V}\right)$. As for the connection Laplacian, the condition (因) implies that $\nabla_{c}$ is a densely-defined, closable operator. Thus, let $\triangleright_{0}$ denote the closure with domain $\mathcal{D}\left(\triangleright_{0}\right)$. It is easy to see that $\nabla_{0} \subset \triangleright$ and $\nabla_{0} \subset \tilde{D}$. We have the following list of equivalences whose proof is similar to that of the analogous proposition we proved for the Laplacians.

Proposition 3.3. Under the same hypotheses as Proposition 3.1, the following are equivalent:

(i) $\triangleright_{0}$ is self-adjoint,

(ii) $\triangleright_{0}=\triangleright=\tilde{\nabla}$,

(iii) $\mathrm{C}_{\mathrm{c}}^{\infty}\left(\mathrm{T}^{*} \mathcal{M} \otimes \mathcal{V}\right)$ is dense in $\mathcal{D}(\triangleright)$,

(iv) $\mathrm{C}_{\mathrm{c}}^{\infty}\left(\mathrm{T}^{*} \mathcal{M} \otimes \mathcal{V}\right)$ is dense in $\mathcal{D}(\tilde{\nabla})$. 
We now prove the main result of this section. We remark that as a consequence of this theorem, each of the statements of Propositions 3.1 3.2, and 3.3 holds.

Theorem 3.4. Let $\mathcal{M}$ be a smooth, complete Riemannian manifold with metric $\mathrm{g}$ and let $\mathcal{V}$ be a smooth vector bundle over $\mathcal{M}$ with smooth metric $\mathrm{h}$ and connection $\nabla$. Suppose that $\mathrm{h}$ and $\nabla$ are compatible. Then, $\overline{\nabla_{c}}=\tilde{\nabla}$ and $\Delta_{0}$ and $\nabla_{0}$ are self-adjoint.

Proof. Let $\mathscr{H}=\mathrm{L}^{2}(\mathcal{V}) \oplus \mathrm{L}^{2}\left(\mathrm{~T}^{*} \mathcal{M} \otimes \mathcal{V}\right)$ and define

$$
\Pi=\left(\begin{array}{cc}
0 & -\operatorname{tr} \nabla \\
\nabla & 0
\end{array}\right)
$$

with domain $\mathcal{D}(\Pi)=\mathrm{C}^{\infty}(\mathcal{V}) \oplus \mathrm{C}^{\infty}\left(\mathrm{T}^{*} \mathcal{M} \otimes \mathcal{V}\right)$. Let $\Pi_{c}$ denote $\Pi$ with domain $\mathcal{D}\left(\Pi_{c}\right)=\mathrm{C}_{\mathrm{c}}^{\infty}(\mathcal{V}) \oplus \mathrm{C}_{\mathrm{c}}^{\infty}\left(\mathrm{T}^{*} \mathcal{M} \otimes \mathcal{V}\right)$. First, we show that $\left\langle\Pi_{c} u, v\right\rangle=\left\langle u, \Pi_{c} v\right\rangle$, for $u, v \in$ $\mathcal{D}\left(\Pi_{c}\right)$. Let $u=\left(u_{1}, u_{2}\right), v=\left(v_{1}, v_{2}\right) \in \mathcal{D}\left(\Pi_{c}\right)$. Then, $\Pi_{c} u=\left(-\operatorname{tr} \nabla_{c} u_{2}, \nabla_{c} u_{1}\right)$ and $\Pi_{c} v=\left(-\operatorname{tr} \nabla_{c} v_{2}, \nabla_{c} v_{1}\right)$. Therefore,

$$
\begin{aligned}
& \left\langle\Pi_{c} u, v\right\rangle=\left\langle-\operatorname{tr} \nabla_{c} u_{2}, v_{1}\right\rangle+\left\langle\nabla_{c} u_{1}, v_{2}\right\rangle \text { and } \\
& \left\langle u, \Pi_{c} v\right\rangle=\left\langle u_{1},-\operatorname{tr} \nabla_{c} v_{2}\right\rangle+\left\langle u_{2}, \nabla_{c} v_{1}\right\rangle .
\end{aligned}
$$

But, by (因),

$$
\left\langle-\operatorname{tr} \nabla_{c} u_{2}, v_{1}\right\rangle=\left\langle u_{2}, \nabla_{c} v_{1}\right\rangle \quad \text { and } \quad\left\langle\nabla_{c} u_{1}, v_{2}\right\rangle=\left\langle u_{1},-\operatorname{tr} \nabla_{c} v_{2}\right\rangle .
$$

Therefore, $\left\langle\Pi_{c} u, v\right\rangle=\left\langle u, \Pi_{c} v\right\rangle$.

Next, we compute the symbol of $\Pi$. Fix $x \in \mathcal{M}$ and $v \in \mathrm{T}_{x}^{*} \mathcal{M}$ and $e=\left(e_{1}, e_{2}\right) \in$ $\mathcal{V}_{x} \oplus \mathrm{T}_{x}^{*} \mathcal{M} \otimes \mathcal{V}_{x}$. Let $f \in \mathrm{C}^{\infty}(\mathcal{V}) \oplus \mathrm{C}^{\infty}\left(\mathrm{T}^{*} \mathcal{M} \otimes \mathcal{V}\right)$ such that $f(x)=e$, and $g \in \mathrm{C}^{\infty}(\mathcal{M})$ such that $\nabla g=v$. Recall the following product rules for $\nabla$ and $\operatorname{tr} \nabla$ :

$$
\nabla(g f)=\nabla g \otimes f+g \nabla f \quad \text { and } \quad \operatorname{tr} \nabla(g f)=g \operatorname{tr} \nabla v+\operatorname{tr}(\overline{\nabla g} \otimes f),
$$

where by $\overline{\nabla g}$ we mean complex conjugation. Write $f=\left(f_{1}, f_{2}\right)$. Then

$$
g \Pi f=g\left(-\operatorname{tr} \nabla f_{2}, \nabla f_{1}\right)
$$

and

$$
\Pi(g f)=\left(-g \operatorname{tr} \nabla f_{2}-\operatorname{tr}\left(\overline{\nabla g} \otimes f_{2}\right), \nabla g \otimes f_{1}+g \nabla f_{1}\right) .
$$

Thus,

$$
\begin{aligned}
\sigma(x, v) e=\Pi(g f)(x)-(g \Pi f)(x) \\
\quad=\left(-\operatorname{tr}\left(\overline{\nabla g(x)} \otimes f_{2}(x)\right), \nabla g(x) \otimes f_{1}(x)\right)=\left(-\operatorname{tr}\left(\bar{v} \otimes e_{2}\right), v \otimes e_{1}\right)
\end{aligned}
$$

since $f(x)=\left(e_{1}, e_{2}\right)$. Therefore,

$$
\begin{aligned}
|\sigma(x, v) e|^{2}=\left|-\operatorname{tr}\left(\bar{v} \otimes e_{2}\right)\right|^{2}+\left|v \otimes e_{1}\right|^{2} \leq|v|^{2}\left|e_{2}\right|^{2}+|v|^{2}\left|e_{1}\right|^{2} & \\
& =|v|^{2}\left(\left|e_{2}\right|^{2}+\left|e_{1}\right|^{2}\right)=|v|^{2}|e|^{2} .
\end{aligned}
$$

From this, it is easy to see that $|\sigma(x, v)|=|v|$, making $\mathrm{c}(x)=1$.

These facts demonstrate that the hypotheses of Theorem 2.3 are satisfied for $\Pi$, and so we conclude that $\Pi_{c}$ is essentially self-adjoint. Letting $\Pi_{0}$ denote the closure of $\Pi_{c}$, note that

$$
\Pi_{0}=\left(\begin{array}{cc}
0 & -\overline{\operatorname{tr} \nabla_{c}} \\
\overline{\nabla_{c}} & 0
\end{array}\right) \quad \text { and } \quad \Pi_{c}^{*}=\left(\begin{array}{cc}
0 & -\operatorname{div} \\
\tilde{\nabla} & 0
\end{array}\right) .
$$


By the self-adjointness of $\Pi_{0}$, we find that $\Pi_{c}^{*}={\overline{\Pi_{0}}}^{*}=\Pi_{0}{ }^{*}=\Pi_{0}$. Thus, $\overline{\nabla_{c}}=\tilde{\nabla}$ and $\overline{\operatorname{tr} \nabla_{c}}=$ div.

Also, we have that

$$
\Pi_{c}^{2}=\left(\begin{array}{cc}
-\operatorname{tr} \nabla_{c}^{2} & 0 \\
0 & -\nabla_{c} \operatorname{tr} \nabla_{c}
\end{array}\right)=\left(\begin{array}{cc}
\Delta_{c} & 0 \\
0 & D_{c}
\end{array}\right)
$$

By Theorem 2.3, $\Pi_{c}^{2}$ is essentially self-adjoint, which implies that $\overline{\Delta_{c}}=\Delta_{0}$ and $\overline{\triangleright_{c}}=\triangleright_{0}$ are also self-adjoint.

Remark 3.5. When $\mathcal{V}$ is $\mathcal{M} \times \mathbb{C}$ (so that the sections of this bundle are measurable functions), the self-adjointness of $\Delta_{0}$ on $\mathrm{L}^{2}(\mathcal{M})$ under the assumption that $\mathcal{M}$ is complete is a well known fact. It was stated in [5] as an application, and here we point to [6] by Gaffney and [10] by Roelcke as historical references.

Denote the $(p, q)$ tensors on $\mathcal{M}$ by $\mathcal{T}^{(p, q)} \mathcal{M}$, where $p$ is the contravariant index and $q$ the covariant index. We point out the following immediate consequence of this theorem for the tensor Laplacian and co-Laplacian.

Corollary 3.6. Let $\mathcal{M}$ be a smooth, complete Riemannian manifold with metric $\mathrm{g}$ and connection $\nabla$ that are compatible. Then, $\mathrm{C}_{\mathrm{c}}^{\infty}\left(\mathcal{T}^{(p, q)} \mathcal{M}\right)$ is dense in $\mathcal{D}\left(\Delta_{\mathrm{B}}\right)$ and $\mathrm{C}_{\mathrm{c}}^{\infty}\left(\mathcal{T}^{(p, q+1)} \mathcal{M}\right)$ is dense in $\mathcal{D}(\triangleright)$.

Remark 3.7. We emphasise that in the corollary, we do not rule out the possibility that $\nabla$ has torsion. That is, the relationship $\nabla_{X} Y-\nabla_{Y} X=[X, Y]$ (where $[\cdot, \cdot]$ denotes the Lie derivative) may fail in general for some $X, Y \in \mathrm{C}^{\infty}(\mathrm{TM})$.

Next, we show some consequences of Theorem 3.4 to Sobolev spaces on vector bundles. Fix $k \in \mathbb{N}$ (although we deal only with $k=1,2$ ). Let $S_{k}$ be the set of $u \in \mathrm{C}^{\infty}(\mathcal{V}) \cap \mathrm{L}^{2}(\mathcal{V})$ such that $\nabla^{i} u \in \mathrm{C}^{\infty}(\mathcal{V}) \cap \mathrm{L}^{2}\left(\mathcal{T}^{(0, i)} \mathcal{M} \otimes \mathcal{V}\right)$ for $1 \leq i \leq k$. Then, the Sobolev norm is defined as $\|u\|_{\mathrm{W}^{k, 2}}=\|u\|+\sum_{i=1}^{k}\left\|\nabla^{i} u\right\|$ for $u \in S_{k}$. We define the Sobolev spaces $\mathrm{W}^{k, 2}(\mathcal{V})$ as the completion of $S_{k}$ under this norm. The Sobolev spaces $\mathrm{W}_{0}^{k, 2}(\mathcal{V})$ are defined as the completion of $\mathrm{C}_{\mathrm{c}}^{\infty}(\mathcal{V})$ under the same norm.

Let $\nabla_{2}: S_{1} \rightarrow \mathrm{C}^{\infty} \cap \mathrm{L}^{2}\left(\mathrm{~T}^{*} \mathcal{M} \otimes \mathcal{V}\right)$ be defined by $\nabla_{2}=\nabla$. Then, we have the following operator theoretic characterisations of $\mathrm{W}^{1,2}(\mathcal{V})$ and $\mathrm{W}_{0}^{1,2}(\mathcal{V})$.

Corollary 3.8. Under the same hypotheses as Theorem 3.4, $\nabla_{2}$ is a denselydefined, closable operator and $\mathrm{W}^{1,2}(\mathcal{V})=\mathcal{D}\left(\overline{\nabla_{2}}\right)$. Furthermore, $\mathrm{W}_{0}^{1,2}(\mathcal{V})=\mathcal{D}\left(\overline{\nabla_{c}}\right)$ and $\mathrm{W}_{0}^{1,2}(\mathcal{V})=\mathrm{W}^{1,2}(\mathcal{V})$.

Proof. Fix $u \in S_{1}$ and $v \in \mathrm{C}_{\mathrm{c}}^{\infty}\left(\mathrm{T}^{*} \mathcal{M} \otimes \mathcal{V}\right)$. Since $x \mapsto \mathrm{h}(u(x),-\operatorname{tr} \nabla v(x))$ is compactly supported, an argument similar to that of the proof of Proposition 6.1 in [4] shows that $\left\langle\nabla_{2} u, v\right\rangle=\langle u,-\operatorname{tr} \nabla v\rangle$. This shows that $\nabla_{2}$ is densely-defined and closable. Furthermore, it shows that $\overline{\nabla_{2}} \subset \tilde{\nabla}$, and it is easy to see that $\overline{\nabla_{c}} \subset \overline{\nabla_{2}}$. Then by Theorem 3.4, we have that $\overline{\nabla_{c}}=\tilde{\nabla}=\overline{\nabla_{2}}$.

Since $\nabla_{2}=\nabla$ on $S_{1}$, it is easy to see that $\mathrm{W}^{1,2}(\mathcal{V})=\mathcal{D}\left(\overline{\nabla_{2}}\right)$. We find that $\mathrm{W}_{0}^{1,2}(\mathcal{V})=\mathcal{D}\left(\overline{\nabla_{c}}\right)$ for a similar reason, and this concludes the proof.

Remark 3.9. (i) In this generality, do not know whether $\mathrm{W}_{0}^{k, 2}(\mathcal{V})=\mathrm{W}^{k, 2}(\mathcal{V})$ for $k \geq 2$. In fact, even for the case $\mathcal{V}=\mathcal{M} \times \mathbb{C}$ with $\nabla$ being the Levi-Cevita connection, we use assumptions on curvature to show that $\mathrm{W}_{0}^{2,2}(\mathcal{M})=\mathrm{W}^{2,2}(\mathcal{M})$. We deal with this situation in \$5 The case for $k>2$ is considered in Proposition 3.2 in [7]. 
(ii) A more general version of this result is true. An alternative argument can be used to dispense the compatibility condition. See Proposition 2.3 in [4].

Next, define $\Xi_{k}: S_{k} \rightarrow \bigoplus_{i=1}^{k} \mathrm{C}^{\infty} \cap \mathrm{L}^{2}\left(\mathcal{T}^{(0, i)} \mathcal{M} \otimes \mathcal{V}\right)$ by $\Xi_{k} u=\left(\nabla^{i} u\right)_{i=1}^{k}$. The following proposition characterises Sobolev spaces in terms of this operator.

Proposition 3.10. Under the same hypothesis as Theorem 3.4, the operator $\Xi_{k}$ is closable and $\mathrm{W}^{k, 2}(\mathcal{V})=\mathcal{D}\left(\overline{\Xi_{k}}\right)$.

Proof. We note that the case $k=1$ is handled in Corollary 3.8 .

Let $u_{n} \in S_{k}$ such that $u_{n} \rightarrow 0$ and $\Xi_{k} u_{n} \rightarrow\left(v_{i}\right)_{i=1}^{k}$. Then, we have that $v_{1}=0$ by Corollary 3.8. Set $w_{n}=\nabla u_{n}$ so that $w_{n} \rightarrow v_{1}=0$ and $\nabla w_{n} \rightarrow v_{2}$. Again, by invoking Corollary [3.8, we conclude that $v_{2}=0$. Proceeding this way, we find that $v_{i}=0$ for all $i=1, \ldots, k$. Thus, $\Xi_{k}$ is closable.

That $\mathrm{W}^{k, 2}(\mathcal{V})=\mathcal{D}\left(\overline{\Xi_{k}}\right)$ follows from the fact that $\|u\|_{\mathrm{W}^{k, 2}}=\|u\|_{\Xi_{k}}=\|u\|+$ $\left\|\Xi_{k} u\right\|$ for $u \in S_{k}$.

We conclude this section with the following regularity result that we use in $\$ 5$ by specialising $\mathcal{V}$ to $\mathcal{M} \times \mathbb{C}$.

Proposition 3.11. Let $\mathcal{M}$ be a smooth, complete Riemannian manifold and $\mathcal{V}$ a smooth vector bundle with metric $\mathrm{h}$ and connection $\nabla$ that are compatible. Then, $\mathrm{W}_{0}^{2,2} \subset \mathcal{D}\left(\Delta_{\mathrm{B}}\right)$ and $\mathrm{W}^{2,2}(\mathcal{V}) \subset \mathcal{D}\left(\Delta_{\mathrm{B}}\right)$ continuously.

Proof. First, we show that whenever $u \in \mathrm{C}^{\infty} \cap \mathrm{L}^{2}(\mathcal{V})$ and $\operatorname{tr} \nabla^{2} u \in \mathrm{C}^{\infty} \cap \mathrm{L}^{2}(\mathcal{V})$, then $u \in \mathcal{D}\left(\Delta_{\mathrm{B}}\right)$ and $\Delta_{\mathrm{B}} u=-\operatorname{tr} \nabla^{2} u$. For that, fix $v \in \mathrm{C}_{\mathrm{c}}^{\infty}(\mathcal{V})$ and let $w \in$ $\mathrm{C}^{\infty} \cap \mathrm{L}^{2}\left(\mathrm{~T}^{*} \mathcal{M} \otimes \mathcal{V}\right)$ with $\operatorname{tr} \nabla w \in \mathrm{C}^{\infty} \cap \mathrm{L}^{2}(\mathcal{V})$. Then, since $x \mapsto \mathrm{h}(v(x),-\operatorname{tr} \nabla w(x))$ is compactly supported, we have $\langle-\operatorname{tr} \nabla w, v\rangle=\left\langle w, \nabla_{c} v\right\rangle$. Furthermore, by an application of the Cauchy-Schwarz inequality, we find that $\nabla u \in \mathrm{C}^{\infty} \cap \mathrm{L}^{2}\left(\mathrm{~T}^{*} \mathcal{M} \otimes \mathcal{V}\right)$. Thus, upon putting $w=\nabla u$, we have that $\left\langle-\operatorname{tr} \nabla^{2} u, v\right\rangle=\left\langle\nabla u, \nabla_{c} v\right\rangle$. Again, since $x \mapsto \mathrm{g}\left(\nabla u(x), \nabla_{c} v(x)\right)_{x}$ is compactly supported, we have that $\left\langle\nabla u, \nabla_{c} v\right\rangle=$ $\left\langle u,-\operatorname{tr} \nabla_{c}^{2} v\right\rangle$. In particular, for our choice of $v$, we have that $\Delta_{\mathrm{B}} v=-\operatorname{tr} \nabla_{c}^{2} v$, and since $\Delta_{\mathrm{B}}$ is self-adjoint, $u \in \mathcal{D}\left(\Delta_{\mathrm{B}}\right)$ and $\Delta_{\mathrm{B}} u=-\operatorname{tr} \nabla^{2} u$.

Now, we show that $\mathrm{W}^{2,2}(\mathcal{V}) \subset \mathcal{D}\left(\Delta_{B}\right)$ continuously. So, fix $u \in \mathrm{W}^{2,2}(\mathcal{V})$ and note that as a consequence of Proposition 3.10, there exists $u_{n} \in \mathrm{C}^{\infty} \cap \mathrm{L}^{2}(\mathcal{V})$ such that $u_{n} \rightarrow u$, and $\Xi_{2} u_{n} \rightarrow \overline{\Xi_{2}} u$. In particular, $\left(\Xi_{2} u_{n}\right)$ is Cauchy, and therefore $\left\|\nabla^{2} u_{n}-\nabla^{2} u_{m}\right\| \rightarrow 0$. Coupling this observation with the fact that $\left|\operatorname{tr} \nabla^{2} u_{n}\right| \leq$ $\left|\nabla^{2} u_{n}\right|$, and since $\Delta_{\mathrm{B}} u_{n}=-\operatorname{tr} \nabla^{2} u_{n}$, we have that $\Delta_{\mathrm{B}} u_{n} \rightarrow v$. But $\Delta_{\mathrm{B}}$ is closed, and hence $u \in \mathcal{D}\left(\Delta_{\mathrm{B}}\right)$ and $v=\Delta_{\mathrm{B}} u$. The continuity of the embedding follows easily.

Since $\mathrm{W}_{0}^{2,2}(\mathcal{V}) \subset \mathrm{W}^{2,2}(\mathcal{V})$ is a continuous embedding, we conclude that $\mathrm{W}_{0}^{2,2}(\mathcal{V})$ $\subset \mathcal{D}\left(\Delta_{\mathrm{B}}\right)$ continuously.

\section{EXterior, INTERIOR DERIVATIVES AND THE DiRAC OPERATOR}

In this section, we consider the density problem for some natural operators over the exterior algebra of $\mathcal{M}$. To fix notation, let the space of $p$-forms on $\mathcal{M}$ be denoted by $\boldsymbol{\Omega}^{p}(\mathcal{M})$. Let the fibre of the bundle $\boldsymbol{\Omega}^{p}(\mathcal{M})$ over $x \in \mathcal{M}$ be denoted by $\boldsymbol{\Omega}_{x}^{p}(\mathcal{M})$. Without causing too much confusion, let us denote the canonical extension of the metric g on $\mathcal{M}$ to $\boldsymbol{\Omega}^{p}(\mathcal{M})$ again by g. The exterior product of a $p$-form $\omega$ and $q$-form $\eta$ is denoted by $\omega \wedge \eta \in \Omega^{p+q}(\mathcal{M})$, and the interior or cut product by 
$\omega\left\llcorner\eta \in \boldsymbol{\Omega}^{q-p}(\mathcal{M})\right.$, which is defined as the dual to $\wedge$. The exterior algebra is then denoted by $\boldsymbol{\Omega}(\mathcal{M})=\bigoplus_{p=0}^{n} \Omega^{p}(\mathcal{M})$. This is a graded algebra with respect to $\wedge$. By $\boldsymbol{\Omega}_{x}(\mathcal{M})$, we denote the fibre of $\boldsymbol{\Omega}(\mathcal{M})$ over $x$.

The exterior and interior derivatives are then defined by the local expressions

$$
\mathrm{d}_{\infty} \omega=d x^{i} \wedge \nabla_{\partial_{i}} \omega \quad \text { and } \quad \delta_{\infty} \omega=-d x^{i}\left\llcorner\nabla_{\partial_{i}} \omega\right.
$$

with domains $\mathcal{D}\left(\mathrm{d}_{\infty}\right)=\mathcal{D}\left(\delta_{\infty}\right)=\mathrm{C}^{\infty}(\boldsymbol{\Omega}(\mathcal{M}))$. On $p$-forms, $\mathrm{d}_{\infty}: \mathrm{C}^{\infty}\left(\boldsymbol{\Omega}^{p}(\mathcal{M})\right) \rightarrow$ $\mathrm{C}^{\infty}\left(\boldsymbol{\Omega}^{p+1}(\mathcal{M})\right)$ and $\delta_{\infty}: \mathrm{C}^{\infty}\left(\boldsymbol{\Omega}^{p}(\mathcal{M})\right) \rightarrow \mathrm{C}^{\infty}\left(\boldsymbol{\Omega}^{p-1}(\mathcal{M})\right)$. By $\mathrm{d}_{c}$ and $\delta_{c}$, denote the operators $\mathrm{d}_{\infty}$ and $\delta_{\infty}$ but with $\mathcal{D}\left(\mathrm{d}_{c}\right)=\mathcal{D}\left(\delta_{c}\right)=\mathrm{C}_{\mathrm{c}}^{\infty}(\boldsymbol{\Omega}(\mathcal{M}))$. Recall also that

$$
\left\langle\mathrm{d}_{c} \omega, \eta\right\rangle=\left\langle\omega, \delta_{c} \eta\right\rangle
$$

for $\omega, \eta \in \mathrm{C}_{\mathrm{c}}^{\infty}(\boldsymbol{\Omega}(\mathcal{M}))$. Thus, $\mathrm{d}_{c}$ and $\delta_{c}$ are densely-defined, closable operators. We denote the closures by $\mathrm{d}_{0}$ and $\delta_{0}$ respectively. Also, let $\mathrm{d}=\delta_{c}^{*}$ and $\delta=\mathrm{d}_{c}^{*}$. Then we have the following theorem.

Theorem 4.1. Let $\mathcal{M}$ be a smooth, complete Riemannian manifold with smooth metric $\mathrm{g}$ and Levi-Cevita connection $\nabla$. Then, $\mathrm{d}_{0}=\mathrm{d}$ and $\delta_{0}=\delta$. In other words, $\mathrm{C}_{\mathrm{c}}^{\infty}(\boldsymbol{\Omega}(\mathcal{M}))$ is dense in $\mathcal{D}(\mathrm{d})$ and in $\mathcal{D}(\delta)$. Furthermore, $\mathrm{C}_{\mathrm{c}}^{\infty}(\boldsymbol{\Omega}(\mathcal{M}))$ is dense in $\mathcal{D}(\mathrm{d} \delta)$ and in $\mathcal{D}(\delta \mathrm{d})$.

Proof. Define

$$
\Pi_{\infty}=\left(\begin{array}{cc}
0 & \delta_{\infty} \\
\mathrm{d}_{\infty} & 0
\end{array}\right)
$$

with $\mathcal{D}\left(\Pi_{\infty}\right)=\mathrm{C}^{\infty}(\boldsymbol{\Omega}(\mathcal{M})) \oplus \mathrm{C}^{\infty}(\boldsymbol{\Omega}(\mathcal{M}))$. Then, let $\Pi_{c}$ be the operator $\Pi_{\infty}$ with $\mathcal{D}(\Pi)=\mathrm{C}_{\mathrm{c}}^{\infty}(\boldsymbol{\Omega}(\mathcal{M})) \oplus \mathrm{C}_{\mathrm{c}}^{\infty}(\boldsymbol{\Omega}(\mathcal{M}))$.

First, we show that $\Pi_{c}$ is symmetric. Fix $u=\left(u_{1}, u_{2}\right), v=\left(v_{1}, v_{2}\right) \in \mathrm{C}_{\mathrm{c}}^{\infty}(\boldsymbol{\Omega}(\mathcal{M}))$, and note that $\Pi_{c} u=\left(\delta_{c} u_{2}, \mathrm{~d}_{c} u_{1}\right), \Pi_{c} v=\left(\delta_{c} v_{2}, \mathrm{~d}_{c} v_{1}\right)$. Then,

$$
\left\langle\Pi_{c} u, v\right\rangle=\left\langle\delta_{c} u_{2}, v_{1}\right\rangle+\left\langle\mathrm{d}_{c} u_{1}, v_{2}\right\rangle=\left\langle u_{2}, \mathrm{~d}_{c} v_{1}\right\rangle+\left\langle u_{1}, \delta_{c} v_{1}\right\rangle=\left\langle u, \Pi_{c} v\right\rangle .
$$

Now, fix $v \in \mathrm{T}^{*} \mathcal{M}$ and $e \in \boldsymbol{\Omega}_{x}(\mathcal{M})$. Let $g \in \mathrm{C}^{\infty}(\mathcal{M})$ and $f \in \mathrm{C}^{\infty}(\boldsymbol{\Omega}(\mathcal{M})) \oplus$ $\mathrm{C}^{\infty}(\boldsymbol{\Omega}(\mathcal{M}))$ such that $\mathrm{d} g(x)=v$ and $f=\left(f_{1}, f_{2}\right)$ and $f(x)=e=\left(e_{1}, e_{2}\right)$. Then,

$\sigma(x, v) e=\left[\Pi_{\infty}, g I\right] f(x)=\Pi_{\infty}(g f)-g \Pi_{\infty} f=\left(\delta_{\infty}\left(g f_{2}\right)-g \delta_{\infty} f_{2}, \mathrm{~d}_{\infty}\left(g f_{1}\right)-g \mathrm{~d}_{\infty} f_{1}\right)$.

Note then that

$$
\mathrm{d}_{\infty}\left(g f_{1}\right)-g \mathrm{~d}_{\infty} f_{1}=\left(\mathrm{d}_{\infty} g \wedge f_{1}+g \mathrm{~d}_{\infty} f_{1}\right)-g \mathrm{~d}_{\infty} f_{1}=\mathrm{d}_{\infty} g \wedge f_{1} .
$$

Thus, at $x, \mathrm{~d}_{\infty} g \wedge f_{1}=v \wedge e_{1}$. By a similar calculation,

$$
\delta_{\infty}\left(g f_{2}\right)-g \delta_{\infty} f_{2}=\left(g \delta_{\infty} f_{2}-\mathrm{d}_{\infty} g\left\llcorner f_{2}\right)-g \delta_{c} f_{2}=-\mathrm{d}_{\infty} g\left\llcorner f_{2}\right.\right.
$$

so that at $x, \mathrm{~d}_{\infty} g\left\llcorner f_{2}=v\left\llcorner e_{2}\right.\right.$. We combine these two calculations so that

$$
|\sigma(x, v) e|^{2}=\left|v \wedge e_{1}\right|^{2}+\mid v\left\llcorner\left. e_{2}\right|^{2} \leq|v|^{2}\left(\left|e_{1}\right|^{2}+\left|e_{2}\right|^{2}\right) \leq|v|^{2}|e|^{2} .\right.
$$

Therefore, $|\sigma(x, v)|=|v|$ and thus $\mathrm{c}(r)=1$. Therefore, by Theorem 2.3, we conclude that $\Pi_{c}$ and the powers of $\Pi_{c}$ are essentially self-adjoint. Thus, $\Pi_{c}^{*}=$ $\bar{\Pi}_{c}^{*}=\overline{\Pi_{c}}$, and since

$$
\Pi_{c}^{*}=\left(\begin{array}{ll}
0 & \delta \\
d & 0
\end{array}\right)
$$

we conclude that $\overline{\mathrm{d}}_{c}=\mathrm{d}$ and $\bar{\delta}_{c}=\delta$. 
Next, note that

$$
\Pi_{c}^{2}=\left(\begin{array}{cc}
\delta_{c} \mathrm{~d}_{c} & 0 \\
0 & \mathrm{~d}_{c} \delta_{c}
\end{array}\right)
$$

and by similar reasoning as above, we have that $\overline{\delta_{c} \mathrm{~d}_{c}}$ and $\overline{\mathrm{d}_{c} \delta_{c}}$ are self-adjoint. It is easy to see that $\overline{\delta_{c} \mathrm{~d}_{c}} \subset \delta \mathrm{d}$ and $\overline{\mathrm{d}_{c} \delta_{c}} \subset \mathrm{d} \delta$. Furthermore, $\delta \mathrm{d}$ and $\mathrm{d} \delta$ are self-adjoint, and so by Proposition 2.1 we conclude that $\overline{\delta_{c} \mathrm{~d}_{c}}=\delta \mathrm{d}$ and $\overline{\mathrm{d}_{c} \delta_{c}}=\mathrm{d} \delta$.

Also, define $\mathrm{D}_{\infty}: \mathrm{C}^{\infty}(\boldsymbol{\Omega}(\mathcal{M})) \rightarrow \mathrm{C}^{\infty}(\boldsymbol{\Omega}(\mathcal{M}))$ by $\mathrm{D}_{\infty}=\mathrm{d}_{\infty}+\delta_{\infty}$ and $\mathrm{D}_{c}=$ $\mathrm{d}_{c}+\delta_{c}$ with domain $\mathcal{D}\left(\mathrm{D}_{c}\right)=\mathrm{C}_{\mathrm{c}}^{\infty}(\boldsymbol{\Omega}(\mathcal{M}))$. An easy calculation shows that

$$
\left\langle\mathrm{D}_{c} \omega, \eta\right\rangle=\left\langle\omega, \mathrm{D}_{c} \eta\right\rangle
$$

when $\omega, \eta \in \mathrm{C}_{\mathrm{c}}^{\infty}(\boldsymbol{\Omega}(\mathcal{M}))$. Thus, $\mathrm{D}_{c}$ is a densely-defined, closable operator. Denote its closure by $\mathrm{D}_{0}$ and let $\mathrm{D}=\mathrm{d}+\delta$. The latter operator is the Hodge-Dirac operator with domain $\mathcal{D}(\mathrm{D})=\mathcal{D}(\mathrm{d}) \cap \mathcal{D}(\delta)$. As a consequence of Theorem 4.1, D is self-adjoint. The Hodge-Laplacian is then denoted by $\mathrm{D}^{2}$. We have the following theorem, which is well known and written as an application in [5], but we include it here for completeness.

Theorem 4.2. Let $\mathcal{M}$ be a smooth, complete Riemannian manifold with smooth metric $\mathrm{g}$ endowed with the Levi-Cevita connection $\nabla$. Then we have that $\mathrm{D}_{0}=\mathrm{D}$ and $\overline{\mathrm{D}_{c}^{2}}=\mathrm{D}^{2}$. In other words, $\mathrm{C}_{\mathrm{c}}^{\infty}(\boldsymbol{\Omega}(\mathcal{M}))$ is dense in $\mathcal{D}(\mathrm{D})$ and in $\mathcal{D}\left(\mathrm{D}^{2}\right)$.

Proof. It is straightforward to check that $\mathrm{D}_{c}$ is symmetric. The computation of the symbol was done in the proof of Theorem 4.1. Explicitly,

$$
\sigma(x, v) e=v \wedge e-v\llcorner e,
$$

and thus $c(r)=1$. Thus, by Theorem 2.3, we have that $\mathrm{D}_{0}$ is self-adjoint. But $\mathrm{D}_{0} \subset \mathrm{D}$ and $\mathrm{D}$ is self-adjoint, so by Proposition 2.1 we have that $\mathrm{D}_{0}=\mathrm{D}$. Similarly, $\overline{\mathrm{D}_{c}^{2}}=\mathrm{D}^{2}$.

\section{Second order Sobolev SPaCes on manifoldS}

Throughout this section, in addition to the smoothness and completeness assumptions on $\mathcal{M}$ and $\mathrm{g}$, we further assume that $\mathcal{M}$ is endowed with the LeviCevita connection $\nabla$. In this setting, we show that $\mathrm{W}_{0}^{2,2}(\mathcal{M})=\mathrm{W}^{2,2}(\mathcal{M})$ whenever Ric $\geq \eta$ g for some $\eta \in \mathbb{R}$. The best currently known result according to Hebey in [7. requires the additional assumption that $\operatorname{inj}(\mathcal{M}) \geq \kappa>0$. This is the content of Proposition 3.3 in [7].

Let us recall some notation from $₫ 3$, but specialised to the case that $\mathcal{V}$ is $\mathcal{M} \times \mathbb{C}$. Let $\nabla_{c}=\nabla$ and $\Delta=-\operatorname{tr} \nabla^{2}: \mathrm{C}^{\infty}(\mathcal{M}) \rightarrow \mathrm{C}^{\infty}(\mathcal{M})$ denote the Laplacian on smooth functions. Furthermore, let $\Delta_{c}=\Delta$ with $\mathcal{D}\left(\Delta_{c}\right)=\mathrm{C}_{\mathrm{c}}^{\infty}(\mathcal{M})$. Since this is a densely-defined, closable operator, let $\Delta_{0}$ be its closure with domain $\mathcal{D}\left(\Delta_{0}\right)$. Recall that the Bochner Laplacian is the self-adjoint operator given by $\Delta_{B}=-\operatorname{div} \overline{\nabla_{c}}$ with domain $\mathcal{D}\left(\Delta_{B}\right)$.

With this notation, we prove the main theorem of this paper.

Proof of Theorem 1.1. We note that the assumptions made in Theorem[1.1] satisfy the hypotheses of Theorem 3.4 and Proposition 3.11 with $\mathcal{V}$ as $\mathcal{M} \times \mathbb{C}$. By the latter result, we have that $\mathrm{W}_{0}^{2,2}(\mathcal{M}) \subset \mathcal{D}\left(\Delta_{\mathrm{B}}\right)$ and $\mathrm{W}^{2,2}(\mathcal{M}) \subset \mathcal{D}\left(\Delta_{\mathrm{B}}\right)$ continuously. 
Next, recall the Bochner-Lichnerowicz-Weitzenböck identity

$$
\langle\nabla \Delta u, \nabla u\rangle=\frac{1}{2} \Delta|\nabla u|^{2}+\left|\nabla^{2} u\right|^{2}+\operatorname{Ric}(\nabla u, \nabla u)
$$

for $u \in \mathrm{C}^{\infty}(\mathcal{M})$. By using the bound Ric $\geq \eta \mathrm{g}$, we obtain that $\left\|\nabla^{2} u\right\| \lesssim$ $\|(I+\Delta) u\|$ whenever $u \in \mathrm{C}_{\mathrm{c}}^{\infty}(\mathcal{M})$. Thus, coupled with the fact that $\Delta_{0}=\Delta_{\mathrm{B}}$ by Theorem 3.4 we find that the reverse inclusion $\mathcal{D}\left(\Delta_{B}\right) \subset \mathrm{W}_{0}^{2,2}(\mathcal{M})$ holds, and this allows us to conclude that $\mathcal{D}\left(\Delta_{B}\right)=\mathrm{W}_{0}^{2,2}(\mathcal{M})$.

Combining these set inclusions, we have that $\mathrm{W}^{2,2}(\mathcal{M}) \subset \mathcal{D}\left(\Delta_{B}\right)=\mathrm{W}_{0}^{2,2}(\mathcal{M})$. But $\mathrm{W}_{0}^{2,2}(\mathcal{M}) \subset \mathrm{W}^{2,2}(\mathcal{M})$ continuously, and so we conclude that $\mathrm{W}_{0}^{2,2}(\mathcal{M})=$ $\mathrm{W}^{2,2}(\mathcal{M})$.

\section{ACKNOWLEDGEMENTS}

Work on this paper commenced whilst the author was visiting Steve Hofmann at the University of Missouri, Columbia, Missouri, supported by an AustralianAmerican Fulbright Scholarship, and was completed while visiting Northwestern University, Evanston, Illinois, supported by that institution. The author wishes to acknowledge these institutions as well as his home institution, the Australian National University.

The author would like to acknowledge and emphasise the invaluable contribution made by Dean Baskin, who introduced the author to the work of Paul Chernoff that made this paper possible. Furthermore, the author would like to thank Andrew Morris and Michael Munn for the many fruitful conversations regarding density problems. The author would also like to acknowledge his Ph.D. supervisor, Alan McIntosh, for his helpful comments, providing corrections, and suggesting improvements to some of the results of this paper.

\section{REFERENCES}

[1] Pascal Auscher, Steve Hofmann, Michael Lacey, Alan McIntosh, and Ph. Tchamitchian, The solution of the Kato square root problem for second order elliptic operators on $\mathbb{R}^{n}$, Ann. of Math. (2) 156 (2002), no. 2, 633-654, DOI 10.2307/3597201. MR1933726 (2004c:47096c)

[2] Andreas Axelsson, Stephen Keith, and Alan McIntosh, The Kato square root problem for mixed boundary value problems, J. London Math. Soc. (2) 74 (2006), no. 1, 113-130, DOI 10.1112/S0024610706022873. MR2254555 (2008m:35078)

[3] Andreas Axelsson, Stephen Keith, and Alan McIntosh, Quadratic estimates and functional calculi of perturbed Dirac operators, Invent. Math. 163 (2006), no. 3, 455-497, DOI 10.1007/s00222-005-0464-x. MR.2207232 (2007k:58029)

[4] Lashi Bandara and Alan McIntosh, The Kato square root problem on vector bundles with generalised bounded geometry, arXiv:1203.0373v2 (2012).

[5] Paul R. Chernoff, Essential self-adjointness of powers of generators of hyperbolic equations, J. Functional Analysis 12 (1973), 401-414. MR0369890 (51 \#6119)

[6] Matthew P. Gaffney, The harmonic operator for exterior differential forms, Proc. Nat. Acad. Sci. U. S. A. 37 (1951), 48-50. MR0048138(13,987b)

[7] Emmanuel Hebey, Nonlinear analysis on manifolds: Sobolev spaces and inequalities, Courant Lecture Notes in Mathematics, vol. 5, New York University, Courant Institute of Mathematical Sciences, New York; Amer. Math. Soc., Providence, RI, 1999. MR 1688256 (2000e:58011)

[8] Tosio Kato, Perturbation theory for linear operators, 2nd ed., Grundlehren der Mathematischen Wissenschaften, Band 132, Springer-Verlag, Berlin, 1976. MR0407617 (53 \#11389)

[9] Andrew J. Morris, The Kato square root problem on submanifolds, J. Lond. Math. Soc. (2) 86 (2012), no. 3, 879-910. MR3000834 
[10] Walter Roelcke, Über den Laplace-Operator auf Riemannschen Mannigfaltigkeiten mit diskontinuierlichen Gruppen (German), Math. Nachr. 21 (1960), 131-149. MR0151927 (27 \#1908)

[11] Kōsaku Yosida, Functional analysis, Classics in Mathematics, Springer-Verlag, Berlin, 1995. Reprint of the sixth (1980) edition. MR.1336382 (96a:46001)

Centre for Mathematics and its Applications, Australian National University, CanBerra, ACT, 0200, Australia

URL: http://maths.anu.edu.au/ pandara

E-mail address: lashi.bandara@anu.edu.au 